\title{
A large-scale retrospective study of the overall survival outcome in nasopharyngeal carcinoma with hypertension in Chinese population
}

\author{
Pei Yang ${ }^{1,2}$, Hesham Elhalawani ${ }^{2}$, Yingrui Shi ${ }^{1}$, Ying Tang $^{3,1}$, Yaqian Han $^{1}$, Yu \\ Zhao $^{1,2}$, Fan Lou ${ }^{1}$ and Hekun Jin ${ }^{1}$ \\ ${ }^{1}$ Department of Head and Neck Radiation Oncology, Hunan Cancer Hospital, Changsha, Hunan, China \\ 2 Department of Radiation Oncology, The University of Texas MD Anderson Cancer Center, Houston, TX, USA \\ ${ }^{3}$ Department of Chinese Traditional Medicine, The Jishou Chinese Traditional Medicine Hospital, Jishou, Hunan, China \\ Correspondence to: Hekun Jin, email: hkjin2008@163.com \\ Keywords: VEGF, NPC, hypertension, radiation theropy, hypoxia \\ Received: March 06, $2017 \quad$ Accepted: April 15, 2017 \\ Published: April 27, 2017 \\ Copyright: Yang et al. This is an open-access article distributed under the terms of the Creative Commons Attribution License 3.0 (CC BY \\ 3.0), which permits unrestricted use, distribution, and reproduction in any medium, provided the original author and source are credited.
}

\section{ABSTRACT}

Background: It is known that hypertension is associated with high levels of vascular endothelial growth factor (VEGF) expression which is, in turn, highly connected to the prognosis of a wide array of cancers. The purpose of this study was to evaluate the relationship between hypertension and prognosis of nasopharyngeal carcinoma (NPC) with definitive radiotherapy in a Chinese population.

Patients and Methods: We retrospectively reviewed 4493 patients with NPC who received definitive radiotherapy from 1995 to 2006, with a minimum follow-up of 5 years. Kaplan-Meier survival analysis and Cox proportional hazard model were utilized to determine the association between hypertension and overall survival (OS).

Results: A total of $\mathbf{8 0 2}$ patients with NPC suffered from hypertension as compared to 3691 patients with no associated hypertension. Kaplan-Meier analysis revealed median overall survival of 101.1 and 110.0 months, respectively $(p<0.05)$. In univariate survival analysis, patients with hypertension had worse $0 S(p<0.05)$ than non-hypertension patients. Patients with higher grade hypertension also had worse OS $(p<0.05)$ compare to patients with grade 1 hypertension. In multivariate survival analysis, patients with hypertension had significantly worse $0 S(p<0.05)$ than nonhypertension patients, as well as $M$ stage $(p<0.001)$, after adjustment for related clinical confounding factors.

Conclusion: Our findings provide evidence that hypertension is an independent factor and result in poorer survival outcomes in patients with NPC, the mechanism is still unclear, and it worth further research.

\section{INTRODUCTION}

Epidemiological evidence shows that high blood pressure is associated with elevated the risk of many kinds of cancers [1-3]. Furthermore, Harding et al. found hypertension, both treated and untreated to be associated with an increased risk of cancer incidence and mortality via pooled collaborative analysis of a large retrospective cohort [4]. Correspondingly, several studies claimed that essential hypertension had increased serum vascular endothelial growth factor (VEGF) levels $[5,6]$. The mechanism was demonstrated that hypertension could cause the microvascular damage and trigger the response of vascular repairing via upregulate the expression of VEGF in plasma [7].

Interestingly, VEGF also known as vascular permeability factor is an important angiogenic agent and endothelial specific mitogen, which has been implicated in the neovascularization and cell proliferation of a wide variety of tumors $[8,9]$. Moreover, the results of the current study show that VEGF expression is significantly increased in patients with histologically advanced NPC, and those showing recurrence and cervical lymph node involvement [10]. Furthermore, high expression of VEGF is also active cancer-related mitogen and highly related to cancer prognosis [11-13]. 
These findings have led to the suggestion that hypertension might have a correlation with survival outcome of cancer. Nasopharyngeal carcinoma (NPC) is a common malignancy in Southeast Asia, especially in the southern coastal area of mainland China, Hong Kong, Macao and Taiwan [14]. Hence, we formulated a study based on the hypothesis that hypertension in patients with NPC may independently influence the survival outcomes of this peculiar disease in a Chinese population.

\section{RESULTS}

Baseline characteristics of subjects are shown in Table 1. The entire sample included 4493 subjects, 2633 patients' dead cause were NPC-related, and 1860 patients were censored including non-cancer related death and track lost. A total of 799 subjects $(17.8 \%)$ were found to be hypertensive, and 3694 (82.2\%) were not before the treatment. Hypertension was more frequently encountered in the cohort of NPC patients older than 46 years of age $(p<0.001)$, and male cohort $(p<0.05)$. The prevalence of smokers and alcohol consumers in the entire cohort was $2505(55.7 \%)$ and $1498(33.4 \%)$, respectively. The other clinical characteristics are well balanced.

Kaplan-Meier analysis showed that patients with hypertension had significantly worse overall survival than that of subjects without hypertension, median survival month is $101.1 \pm 2.9$ and $110 \pm 1.6$, respectively $(p<0.05)$ (Figure 1), as well as the elder patient $(p<0.05)$.

Correspondently, the median survival time are 104.9 $\pm 2.9,67.5 \pm 14.1$, and $50.1 \pm 19.7$ months with grade 1,2 , and 3 , respectively. The patients with grade 2 hypertension had worse overall survival outcome than grade 1 ( $p<$ 0.001 ) (Figure 2). The patients with grade 3 hypertension also had worse OS compare to patients with grade 1 ( $p$ $<0.05$ ) (Figure 3). There are no statistical significant of overall survival between patients with grade 2 and grade 3 hypertension $(p>0.05)$. (Figure 4$)$

Univariate Cox proportional hazards regression analysis was performed to estimate risk factor for clinical outcomes. Results indicate that elder patients $(p<0.05)$, hypertension $(p<0.05)$, and positive metastatic disease $(p<0.001)$ were correlated with increased mortality in patients with NPC (Table 2).

Multivariate Cox proportional hazards regression analysis controlling potential confounders was conducted to evaluate association hypertension and clinical outcomes (Table 3). In this study, multiple analyses indicated that hypertension significantly increases the risk of mortality in patients with NPC after adjustment $(\mathrm{RR}=1.110,95.0 \%$ CI: 1.005-1.225 $P=0.039$ ). (Table 4)

\section{DISCUSSION}

To our knowledge, this is the first study to be conducted to evaluate the extent by which hypertension is associated within Chinese patients with NPC. The main finding was that NPC patients with hypertension have more inferior survival outcomes compared with those without hypertension. Unfortunately, no previous

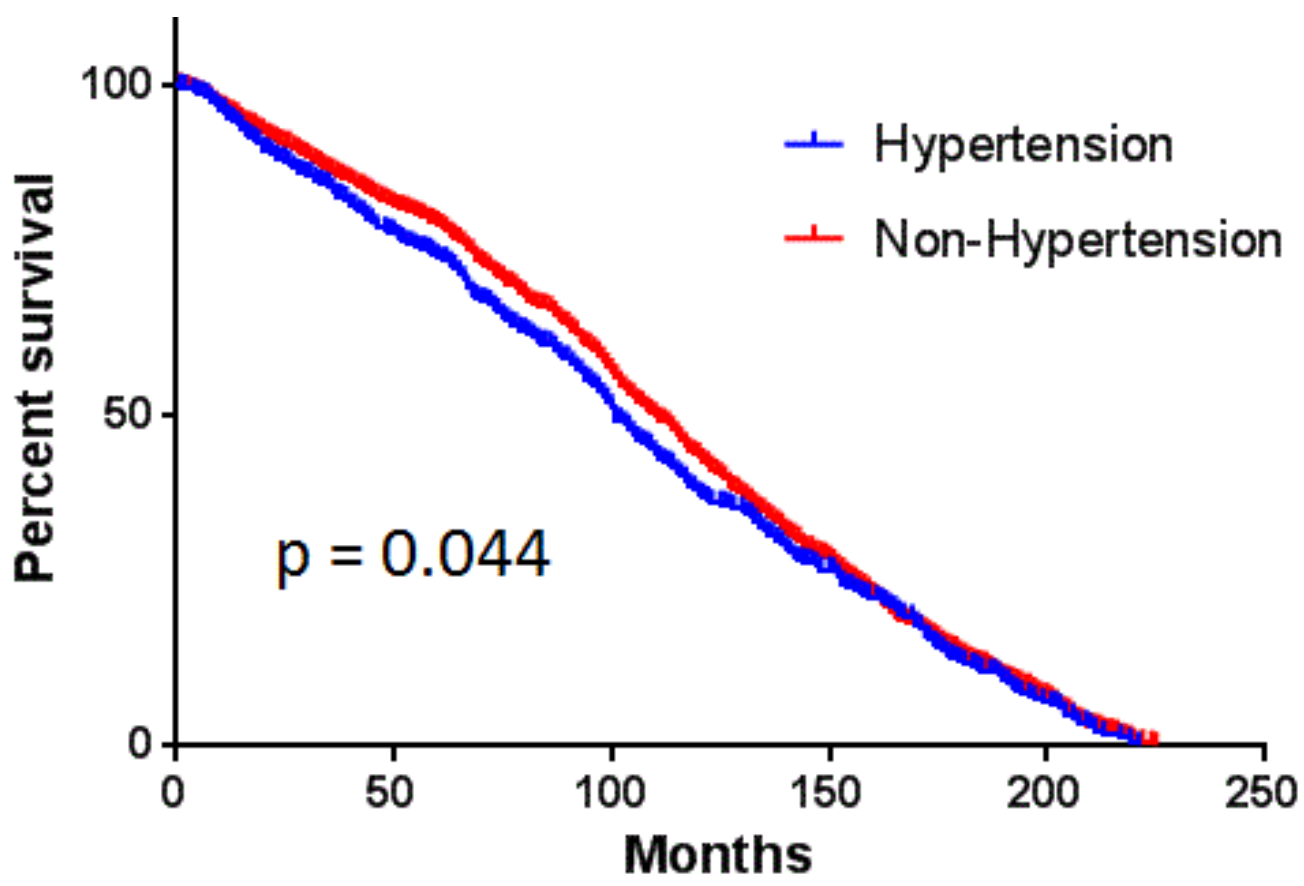

Figure 1: The comparison of overall survival between patients with and without hypertension. 
Table 1: Baseline characteristics between hypertensive and non-hypertensive patients with NPC

\begin{tabular}{|c|c|c|c|}
\hline Variables & Hypertensive & Non-Hypertensive & $p$ \\
\hline & $N(\%)$ & $N(\%)$ & \\
\hline Age & & & 0.000 \\
\hline$<46$ & $311(15.2 \%)$ & $1737(84.8 \%)$ & \\
\hline$\geq 46$ & $491(20.1 \%)$ & $1954(79.9 \%)$ & \\
\hline Gender & & & 0.047 \\
\hline Male & $632(18.5 \%)$ & $2787(81.5 \%)$ & \\
\hline Female & $170(15.8 \%)$ & $904(84.2 \%)$ & \\
\hline KPS & & & 0.712 \\
\hline$\geq 70$ & $784(17.9 \%)$ & $3600(82.1 \%)$ & \\
\hline$<70$ & $18(16.5 \%)$ & $91(83.5 \%)$ & \\
\hline Smoking & & & 0.394 \\
\hline Yes & $458(18.3 \%)$ & $2047(81.7 \%)$ & \\
\hline No & $344(17.3 \%)$ & $1644(82.7 \%)$ & \\
\hline Alcohol & & & 0.297 \\
\hline Yes & $280(18.7 \%)$ & $1218(81.3 \%)$ & \\
\hline No & $522(17.4 \%)$ & $2473(82.6 \%)$ & \\
\hline T Stage & & & 0.126 \\
\hline T0-2 & $435(17.1 \%)$ & $2111(82.9 \%)$ & \\
\hline T3-4 & $367(18.8 \%)$ & $1580(81.2 \%)$ & \\
\hline N Stage & & & 0.395 \\
\hline N0-1 & $427(18.3 \%)$ & $1904(81.7 \%)$ & \\
\hline $\mathrm{N} 2-3$ & $375(17.3 \%)$ & $1787(82.7 \%)$ & \\
\hline M Stage & & & 0.183 \\
\hline M0 & $782(18.0 \%)$ & $3565(82.2 \%)$ & \\
\hline M1 & $20(13.7 \%)$ & $126(86.3 \%)$ & \\
\hline Chemotherapy & & & 0.901 \\
\hline Yes & $659(17.9 \%)$ & $3026(82.1 \%)$ & \\
\hline No & $143(17.7 \%)$ & $665(82.3 \%)$ & \\
\hline Pathology & & & 0.430 \\
\hline Nonkeratinizing & $738(17.7 \%)$ & $3426(82.3 \%)$ & \\
\hline Other & $64(19.5 \%)$ & $265(80.5 \%)$ & \\
\hline
\end{tabular}

*The pathology types of keratinizing and undifferentiated were combined in the other

document has reported to relationships between NPC and elevated blood pressure or hypertension.

In general, upon hypertension-induced microvascular damage, a vast array of growth factors, cytokines, and other molecules are released, stimulating angiogenesis via VEGF, which is essential for the repair process and results in the high level of VEGF expression [7, 15]. Previous studies have also demonstrated that VEGF is increased in hypertensive patients and downregulated after control of blood pressure [5]. Correspondingly, several studies have claimed that essential hypertension patients also have increased serum VEGF levels [5, 6]. VEGF is a multifunctional glycoprotein which is mitogenic for endothelial cells and is the most important regulator of physiological or pathological angiogenesis, the process of new blood vessel growth from preexisting vessels, which is imperative to malignant tumor growth [16]. Many complex molecular pathways that govern tumor angiogenesis are logical targets for pharmacological manipulation given the important role they play in the growth and development of cancers. Along the same lines, VEGF was demonstrated as one of the most important factors in tumor angiogenesis by increasing blood vessel permeability, endothelial cell growth, proliferation, migration, and differentiation [1719]. Moreover, overexpression of VEGF has been linked to tumor progression and poor prognosis in many tumor types [20-22].

Furthermore, the chronic hypoxia causes increased systemic arterial pressure and massive activation of the sympathetic nervous system in healthy humans, that is to say, the patients with hypertension have the hypoxia inner environment exists [23]. Hypoxia itself was reported related to the worse clinical survival outcome 


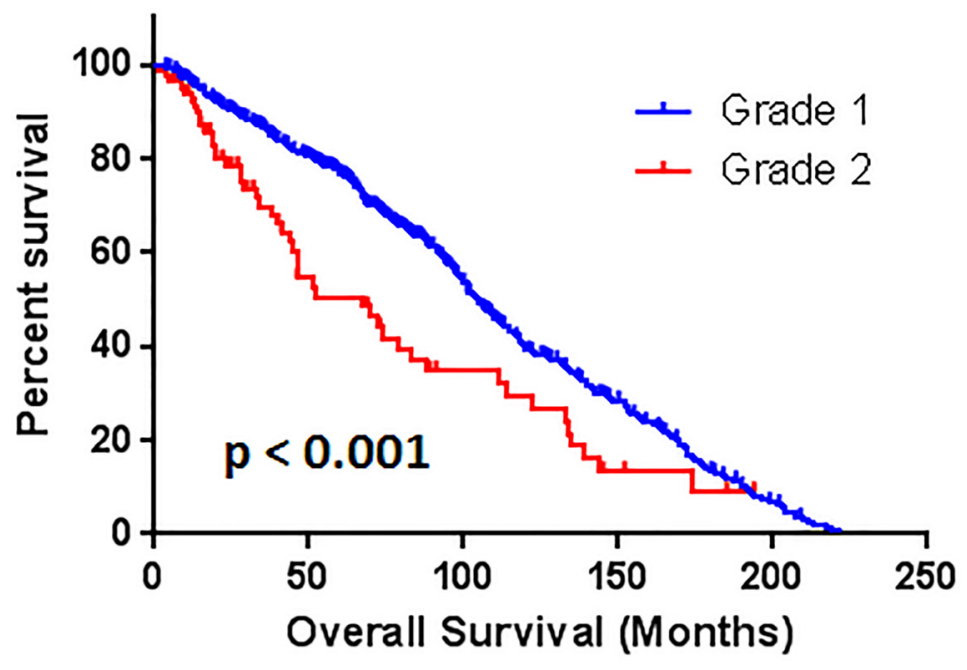

Figure 2: The comparison of overall survival between patients with grade 1 and grade 2.

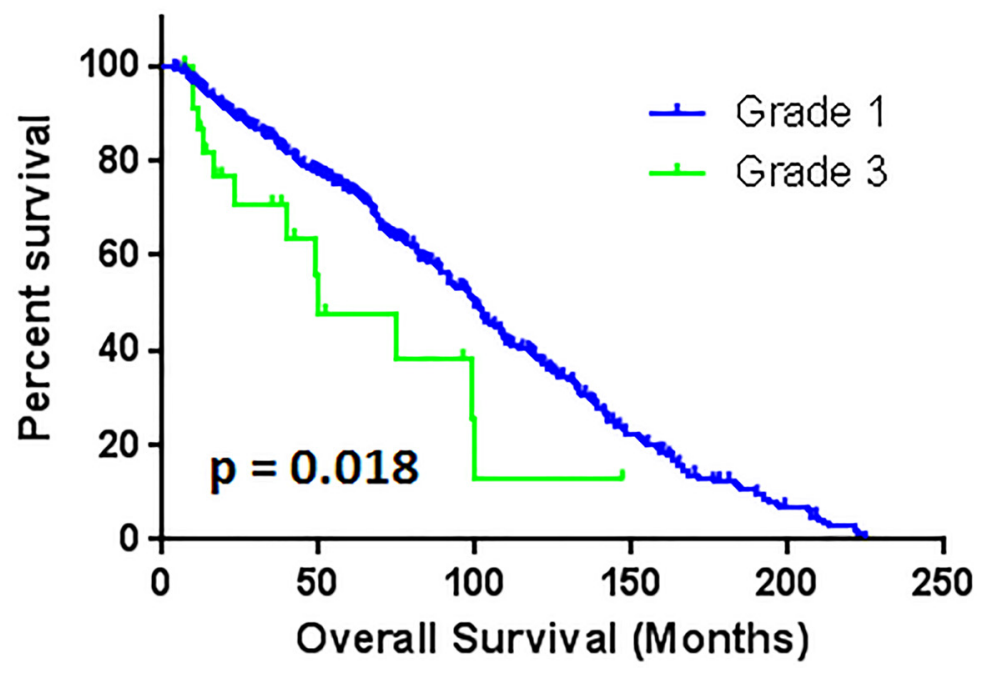

Figure 3: The comparison of overall survival between patients with grade 1 and grade 3.

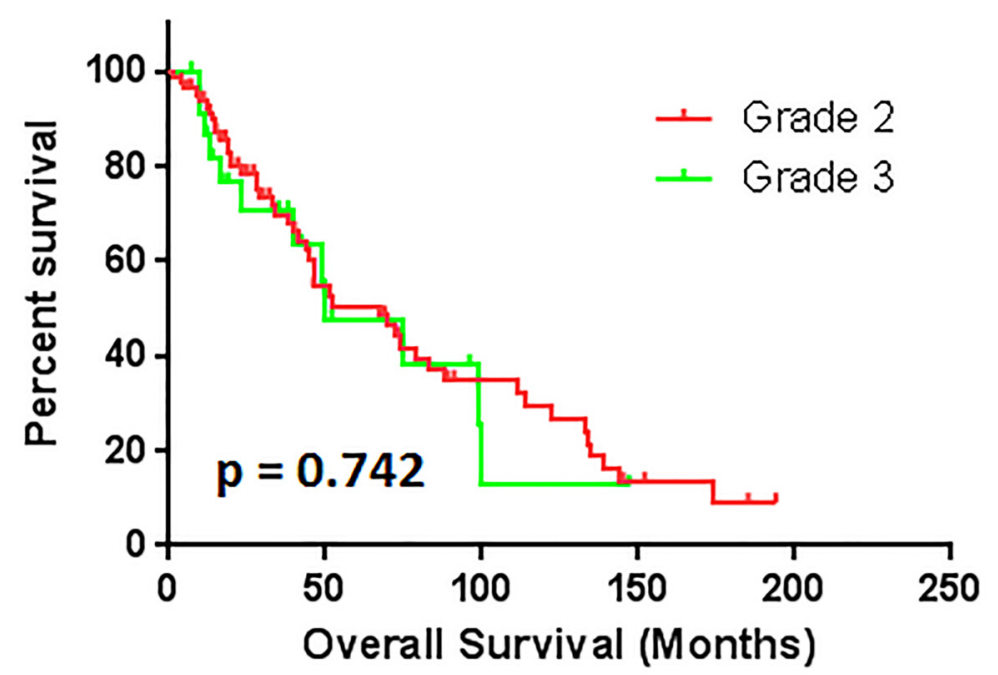

Figure 4: The comparison of overall survival between patients with grade 2 and grade 3. 
Table 2: Uni-variate OS analysis of prognostic factors

\begin{tabular}{|c|c|c|}
\hline \multirow[b]{2}{*}{ Variables } & \multicolumn{2}{|l|}{ OS } \\
\hline & HR (95\% CI) & $p$ \\
\hline \multicolumn{3}{|l|}{ Age } \\
\hline$<66$ & 1.00 & -- \\
\hline$\geq 66$ & $1.042(0.956-1.137)$ & 0.350 \\
\hline \multicolumn{3}{|l|}{ Gender } \\
\hline Male & 1.00 & -- \\
\hline Female & $0.979(0.896-1.069)$ & 0.636 \\
\hline \multicolumn{3}{|l|}{ KPS } \\
\hline$\geq 70$ & 1.00 & -- \\
\hline$<70$ & $1.085(0.768-1.533)$ & 0.644 \\
\hline \multicolumn{3}{|l|}{ Smoking } \\
\hline Yes & 1.00 & -- \\
\hline No & $1.035(0.958-1.118)$ & 0.381 \\
\hline \multicolumn{3}{|l|}{ Alcohol } \\
\hline Yes & 1.00 & -- \\
\hline No & $0.984(0.907-1.068)$ & 0.702 \\
\hline \multicolumn{3}{|l|}{ Hypertension } \\
\hline None & 1.00 & -- \\
\hline Yes & $1.107(1.003-1.222)$ & 0.044 \\
\hline \multicolumn{3}{|l|}{ T stage } \\
\hline$T_{0-2}$ & 1.00 & -- \\
\hline$T_{3-4}$ & $1.059(0.979-1.146)$ & 0.150 \\
\hline \multicolumn{3}{|l|}{ N stage } \\
\hline $\mathbf{N}_{0-1}$ & 1.00 & -- \\
\hline $\mathbf{N}_{2-3}$ & $0.966(1.027-1.197)$ & 0.378 \\
\hline \multicolumn{3}{|l|}{ M stage } \\
\hline $\mathbf{M}_{0}$ & 1.00 & -- \\
\hline $\mathbf{M}_{1}$ & $6.216(4.989-7.745)$ & $<0.001$ \\
\hline \multicolumn{3}{|l|}{ Pathology } \\
\hline Nonkeratinizing & 1.00 & -- \\
\hline Others & $1.083(0.930-1.263)$ & 0.305 \\
\hline \multicolumn{3}{|l|}{ Chemotherapy } \\
\hline Yes & 1.00 & -- \\
\hline No & $0.936(0.848-1.033)$ & 0.188 \\
\hline
\end{tabular}

[24]. The status of hypoxia upgrade the level of VEGF in response to a tumor microenvironment via the hypoxiainducible transcription factor- $1 \alpha(\mathrm{HIF}-1 \alpha)$ and peroxisome proliferator-activated receptor-gamma coactivator (PGC$1 \alpha$ ), Expression of VEGF in response to hypoxia is key to this process and has led to VEGF being defined as the prime hypoxia-inducible angiogenic factor $[25,26]$. Moreover, hypoxia is also an important contributor to tumor radioresistance, the plasma level of HIF-1 decrease the radiosensitivity [27, 28]. However, radiotherapy is the major treatment in NPC patients; low radiosensitivity is strongly associated to the worse prognostic in head and neck cancers $[29,30]$. Several studies also proved the high expression of VEGF-related to the poor survival prognostic in nasopharyngeal carcinoma cancer patients [11-13]. Hence, these connections could be the main reason that NPC patients with high-grade hypertension had worse survival outcome. (Figure 5)

In our findings, elder patients (age $\geq 46$ years) had worse OS $(p<0.05)$, we figure the reason was that the percentage of hypertension distributed higher in the cohort of elder patients. This result is also consistent with several findings [31-33]. Unfortunately, all the researchers failed on taking hypertension as one of the potential factors.

However, there are several limitations in this study. First, the examination on the level of VEGF/HIF1 in plasma of all patients was not available. Secondly, some studies indicate that anti-hypertension medications, 
Table 3: Multi-variate OS analysis of all prognostic factors

\begin{tabular}{|c|c|c|}
\hline \multirow[b]{2}{*}{ Variables } & \multicolumn{2}{|l|}{ OS } \\
\hline & HR (95\% CI) & $p$ \\
\hline \multicolumn{3}{|l|}{ Age } \\
\hline$<46$ & 1.00 & -- \\
\hline$\geq 46$ & $1.089(1.008-1.176)$ & 0.030 \\
\hline \multicolumn{3}{|l|}{ Gender } \\
\hline Male & 1.00 & -- \\
\hline Female & $0.997(0.910-1.091)$ & 0.997 \\
\hline \multicolumn{3}{|l|}{ KPS } \\
\hline$\geq 70$ & 1.0 & -- \\
\hline$<70$ & $1.133(0.801-1.601)$ & 0.480 \\
\hline \multicolumn{3}{|l|}{ Smoking } \\
\hline Yes & 1.00 & -- \\
\hline No & $1.034(0.955-1.118)$ & 0.408 \\
\hline \multicolumn{3}{|l|}{ Alcohol } \\
\hline Yes & 1.00 & -- \\
\hline No & $0.987(0.908-1.073)$ & 0.757 \\
\hline \multicolumn{3}{|l|}{ Hypertension } \\
\hline None & 1.00 & -- \\
\hline Yes & $1.111(1.006-1.227)$ & 0.037 \\
\hline \multicolumn{3}{|l|}{ T stage } \\
\hline$T_{0-2}$ & 1.00 & -- \\
\hline $\mathbf{T}_{3-4}$ & $1.047(0.968-1.133)$ & 0.250 \\
\hline \multicolumn{3}{|l|}{ N stage } \\
\hline $\mathbf{N}_{0-1}$ & 1.00 & -- \\
\hline $\mathbf{N}_{2-3}$ & $0.970(0.898-1.047)$ & 0.430 \\
\hline \multicolumn{3}{|l|}{ M stage } \\
\hline $\mathbf{M}_{0}$ & 1.00 & -- \\
\hline $\mathbf{M}_{0}$ & $6.224(4.993-7.758)$ & $<0.001$ \\
\hline \multicolumn{3}{|l|}{ Pathology } \\
\hline Nonkeratinizing & 1.00 & -- \\
\hline Others & $1.069(0.917-1.246)$ & 0.394 \\
\hline \multicolumn{3}{|l|}{ Chemotherapy } \\
\hline Yes & 1.00 & -- \\
\hline No & $0.926(0.839-1.023)$ & 0.129 \\
\hline
\end{tabular}

Note: The pathology types of keratinizing and undifferentiated were combined in the other; all prognostic factors were analysis and adjusted using enter method in $\mathrm{COX}$

Table 4: Multivariate survival analysis for nasopharyngeal carcinoma (final best model)

\begin{tabular}{|l|c|c|}
\hline \multirow{2}{*}{ Variables } & HR (95\% CI) & $\boldsymbol{p}$ \\
\cline { 2 - 3 } Age & & \\
\hline$<46$ & 1.00 & - \\
\hline$\geq 46$ & $1.087(1.007-1.173)$ & $\mathbf{0 . 0 3 3}$ \\
\hline Hypertension & 1.00 & -- \\
\hline No & $1.110(1.005-1.225)$ & $\mathbf{0 . 0 3 9}$ \\
\hline Yes & & -- \\
\hline $\mathbf{M}_{\text {stage }}$ & 1.00 & $<\mathbf{0 . 0 0 1}$ \\
\hline $\mathbf{M}_{0}$ & $6.257(5.022-7.796)$ & \\
\hline $\mathbf{M}_{0}$ & & \\
\hline
\end{tabular}

Note: All three significant factors in the model via back step method. 
such as beta-blockers, might have the potential effect of reducing the level of VEGF in plasma [34]. Unfortunately, we were unable to retrieve the drug usage in this cohort of patients. Moreover, most of NPC patients have the high expression of Epstein-Barr virus (EBV), and the correlation between VEGF expression and EBV status was explored, where EBV was suggested to have a role in angiogenesis and subsequent disease progression via VEGF upregulation, this mechanism could result in an interactive reinforcement in NPC patients. Unfortunately, the exam of EBV was not performed while this cohort of patients received radiotherapy from 1995 to 2006 [35].

Base on the mechanism above and the limitation of this retrospective study, our group have already been proceeding a relative large-scale prospective research on it.

In conclusion, our findings provide evidence that hypertension is associated with overall survival outcome of NPC patients, along with the positive metastatic disease.
We postulate that the possible mechanism is the higher plasma VEGF level in the patients with hypertension. Although there are several limitations in this study, this finding still worth further research.

\section{MATERIALS AND METHODS}

\section{Study population}

We scanned our medical records of Chinese patients with NPC who were treated with definitive chemoradiotherapy from 1995 to 2006 at Hunan Cancer Hospital. Retrospectively recruited 4493 consecutive Chinese patients. The inclusion criteria were as follows: (a): histopathologically confirmed NPC, (b): receipt of definitive radiotherapy with or without concurrent chemotherapy. (c): no other primary cancer or a history

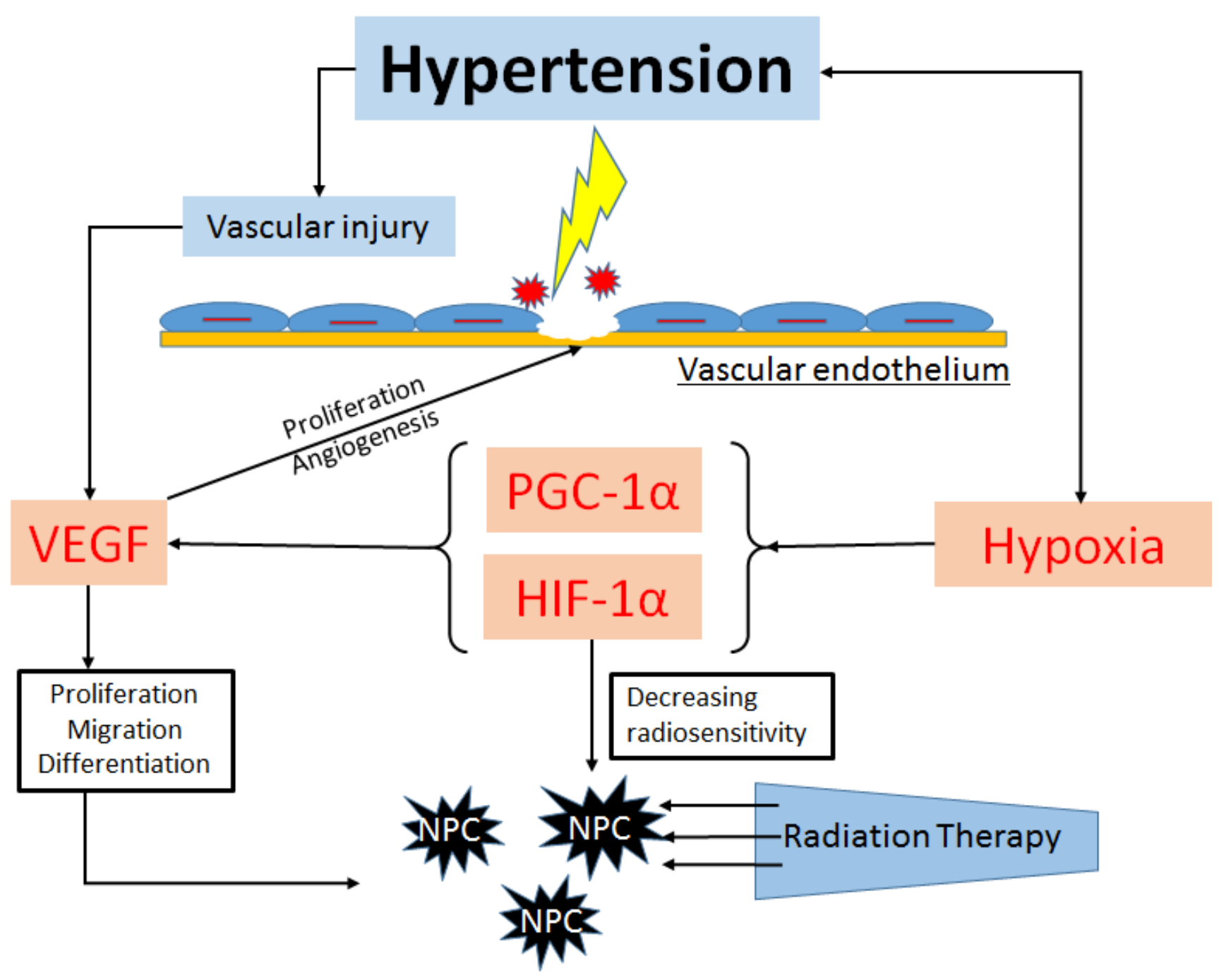

Figure 5: The possible mechanisms of hypertension affect the survival outcome in NPC patients. 
of another cancer. Patients were excluded from the study to eliminate potential confounding factors which may influence the clinical outcome of NPC. Exclusion criteria included the following: 1) history or findings of significant valvular heart disease (i.e. more severe than mild valvular insufficiency or stenosis), hyperthyroidism or hypothyroidism or cardiomyopathy (dilated or hypertrophic); 2) atrial fibrillation; 3) pregnancy; and/ or 4) a major systemic illness such as Systemic lupus erythematosus. A written consent was obtained from all patients before data accrual. Moreover, this study was approved by the Ethics Committee of the Hunan Cancer Hospital, Changsha, China.

\section{Measurement and definition}

This patient database contained detailed demographic data, the patient's status (smoking index, alcohol consumption measurements, etc.), comprehensive tumor details, clinical stage, histological subtype, radiotherapy (RT) data, chemotherapy data, treatment outcomes, and mortality data. The disease was restaged according to the seventh edition of the American Joint Committee on Cancer (AJCC) staging system [36], and the pathological tumor types were determined according to the World Health Organization (WHO) NPC classification [37]. The diagnosis of hypertension was derived from the medical records of every patient. We evaluated and categorized the patient's hypertension grade with the average blood pressure based on the weekly result of blood pressure during the whole treatment in our hospital. The definition of hypertension was according to American Heart Association (AHA) guidelines [38]. All patients underwent definitive RT 5 days per week by using traditional conformal radiotherapy technology.

\section{Clinical endpoints}

At a median follow-up of 80 weeks, all included patients were further contacted by telephone or mail to screen for new death events. The data set was completed by information obtained from relatives, attending physicians and hospital records. In this study, our clinical endpoints included both NPC-related deaths, as well as other non-cancer-related deaths, were also documented.

\section{Statistics analysis}

Differences in the distribution of baseline characteristics between individuals with censor and outcome groups were examined using Chi-square test for categorical variables and Student's $T$-test for continuous variables. Descriptive statistics are presented as percentages. All $P$-values are two-sided, and values of
$<0.05$ were considered to be statistically significant. For clean interpretation and summarization of results, KaplanMeier time-to-event analyses were used for clinical outcomes, with log-rank tests used for differences between previous study-group assignments. Cox proportional hazards regression models adjusted for potential confounders were used to study the relation between relative risk of death and hypertension severity at baseline. First, univariate Cox regression analyses were carried out to examine the association between each potential confounder and clinical outcomes. Potential confounders for outcomes included age, gender, smoking index, alcohol consumption, T, N and $\mathrm{M}$ categories, NPC WHO pathological subtypes, and hypertension grade of severity. Secondly, we fitted separate univariable Cox regression models to evaluate the influence of each covariate in the strength of association between hypertension severity and clinical outcomes. The adjusted hazard ratio of the results among NPC patients with hypertension compared with those without hypertension was the basic model from which the effect of each covariate was estimated. Estimates derived from Cox regressions are presented as hazard ratios and 95\% confidence intervals (CI). Statistical analyses were performed using SPSS version 23.0

\section{Abbreviations}

VEGF: vascular endothelial growth factor ; NPC: Nasopharyngeal carcinoma; RT: radiotherapy; OS: overall survival; HIF-1 $\alpha$ : hypoxia-inducible transcription factor$1 \alpha$; PGC-1 $\alpha$ : peroxisome proliferator-activated receptorgamma coactivator; EBV: Epstein-Barr virus; AJCC: American Joint Committee on Cancer; WHO: World Health Organization; AHA: American Heart Association

\section{Co-author specific contributions}

All listed co-authors performed the following:

1. Substantial contributions to the conception or design of the work; or the acquisition, analysis, or interpretation of data for the work;

2. Drafting the work or revising it critically for important intellectual content;

3. Final approval of the version to be published;

4. Agreement to be accountable for all aspects of the work in ensuring that questions related to the accuracy or integrity of any part of the work are appropriately investigated and resolved.

Specific additional individual cooperative effort contributions to study/manuscript design/execution/ interpretation, in addition to all criteria above, are listed as follows:

- Pei Yang, Hekun Jin, Hesham Elhalawani, Yingrui Shi - Manuscript writing, statistical analysis, clinical data workflows; direct oversight of trainee personnel (Ying 
Tang, Yu Zhao, ).

- Pei Yang, - Statistical analysis, the guarantor(s) of statistical quality, case-matching.

- Ying Tang, Yu Zhao - Electronic medical record screening, automated case identification, data extraction, clinical data collection and informatics software support.

- Yaqian Han, Fan Lou - Database construction, clinical/oncologic database curation and oversight, conceptual feedback and support.

- Hekun Jin- the Corresponding author; primary investigator; conceived, coordinated and directed all study activities, responsible for data collection, project integrity, manuscript content and editorial oversight and correspondence; direct oversight of trainee personnel.

\section{ACKNOWLEDGMENTS}

We thank our colleagues from Hunan cancer hospital (China), MD Anderson Cancer Center (USA), Jishou Chinese traditional medicine Hospital (China), Xiangya medical school, the University of Central South (China), and Shanghai Tongji Hospital (China), who provided their insight and expertise that greatly assisted the research, and they all agree with the interpretations/conclusions of this paper.

\section{CONFLICTS OF INTEREST}

The authors declare no conflicts of interest.

\section{FUNDING}

This research was partially supported by Chinese International Medical Foundation (No: Z-2014-06-15324); Hunan Provincial Health and family planning commission (B2015-109)

\section{REFERENCES}

1. Radisauskas R, Kuzmickiene I, Milinaviciene E, Everatt R. Hypertension, serum lipids and cancer risk: A review of epidemiological evidence. Medicina (Kaunas). 2016; 52: 89-98. doi: 10.1016/j.medici.2016.03.002.

2. Schairer C, Gadalla SM, Pfeiffer RM, Moore SC, Engels EA. Diabetes, Abnormal Glucose, Dyslipidemia, Hypertension and Risk of Inflammatory and Other Breast Cancer. Cancer Epidemiol Biomarkers Prev. 2017; 26:862868. doi: 10.1158/1055-9965.epi-16-0647.

3. Liang Z, Xie B, Li J, Wang X, Wang S, Meng S, Ji A, Zhu Y, Xu X, Zheng X, Xie L. Hypertension and risk of prostate cancer: a systematic review and meta-analysis. Sci Rep. 2016; 6: 31358. doi: 10.1038/srep31358.

4. Harding JL, Sooriyakumaran M, Anstey KJ, Adams R, Balkau B, Brennan-Olsen S, Briffa T, Davis TM, Davis WA, Dobson A, Giles GG, Grant J, Huxley R, et al. Hypertension, antihypertensive treatment and cancer incidence and mortality: a pooled collaborative analysis of 12 Australian and New Zealand cohorts. J Hypertens. 2016; 34: 149-55. doi: 10.1097/hjh.0000000000000770.

5. Belgore FM, Blann AD, Li-Saw-Hee FL, Beevers DG, Lip GY. Plasma levels of vascular endothelial growth factor and its soluble receptor (SFlt-1) in essential hypertension. Am J Cardiol. 2001; 87: 805-7. doi: 10.1016/S00029149(00)01512-5.

6. Ayerden Ebinc F, Haksun E, Ulver DB, Koc E, Erten Y, Reis Altok K, Bali M, Turgay A, Sindel S. The relationship between vascular endothelial growth factor (VEGF) and microalbuminuria in patients with essential hypertension. Intern Med. 2008; 47: 1511-6. doi: 10.2169/ internalmedicine.47.1122.

7. Tsai WC, Li YH, Huang YY, Lin CC, Chao TH, Chen JH. Plasma vascular endothelial growth factor as a marker for early vascular damage in hypertension. Clin Sci (Lond). 2005; 109: 39-43. doi: 10.1042/cs20040307.

8. Ajani J, Allgood V. Molecular mechanisms in cancer: what should clinicians know? Semin Oncol. 2005; 32: 2-4. doi: 10.1053/j.seminoncol.2005.07.016.

9. Yoshiji H, Gomez DE, Shibuya M, Thorgeirsson UP. Expression of vascular endothelial growth factor, its receptor, and other angiogenic factors in human breast cancer. Cancer Res. 1996; 56: 2013-6.

10. Sun R, Wang X, Li X. Correlation Analysis of Nasopharyngeal Carcinoma TNM Staging with Serum EA IgA and VCA IgA in EBV and VEGF-C and -D. Med Sci Monit. 2015; 21: 2105-9. doi: 10.12659/msm.893415.

11. Zhang JX, Cai MB, Wang XP, Duan LP, Shao Q, Tong ZT, Liao DZ, Li YY, Huang MY, Zeng YX, Shao JY. Elevated DLL4 expression is correlated with VEGF and predicts poor prognosis of nasopharyngeal carcinoma. Med Oncol. 2013; 30: 390. doi: 10.1007/s12032-012-0390-x.

12. Kim TJ, Lee YS, Kang JH, Kim YS, Kang CS. Prognostic significance of expression of VEGF and Cox2 in nasopharyngeal carcinoma and its association with expression of C-erbB2 and EGFR. J Surg Oncol. 2011; 103: 46-52. doi: 10.1002/jso.21767.

13. Pan J, Tang T, Xu L, Lu JJ, Lin S, Qiu S, Chen G, K Tham IW. Prognostic significance of expression of cyclooxygenase-2, vascular endothelial growth factor, and epidermal growth factor receptor in nasopharyngeal carcinoma. Head Neck. 2013; 35: 1238-47. doi: 10.1002/ hed.23116.

14. Chang ET, Adami HO. The enigmatic epidemiology of nasopharyngeal carcinoma. Cancer Epidemiol Biomarkers Prev. 2006; 15: 1765-77. doi: 10.1158/1055-9965.epi-060353.

15. Felmeden DC, Blann AD, Lip GY. Angiogenesis: basic pathophysiology and implications for disease. Eur Heart J. 2003; 24: 586-603. doi: 10.1016/S0195-668X(02)00635-8.

16. Ferrara N. Role of vascular endothelial growth factor in the 
regulation of angiogenesis. Kidney Int. 1999; 56: 794-814. doi: 10.1046/j.1523-1755.1999.00610.x.

17. Hicklin DJ, Ellis LM. Role of the vascular endothelial growth factor pathway in tumor growth and angiogenesis. J Clin Oncol. 2005; 23: 1011-27. doi: 10.1200/ jco.2005.06.081.

18. Ferrara N. The role of vascular endothelial growth factor in pathological angiogenesis. Breast Cancer Res Treat. 1995; 36: 127-37. doi: 10.1007/BF00666035.

19. Sakurai T, Kudo M. Signaling pathways governing tumor angiogenesis. Oncology. 2011; 81: 24-9. doi: 10.1159/000333256.

20. Loncaster JA, Cooper RA, Logue JP, Davidson SE, Hunter $\mathrm{RD}$, West CM. Vascular endothelial growth factor (VEGF) expression is a prognostic factor for radiotherapy outcome in advanced carcinoma of the cervix. Br J Cancer. 2000; 83: 620-5. doi: 10.1054/bjoc.2000.1319.

21. Lee YH, Tokunaga T, Oshika Y, Suto R, Yanagisawa K, Tomisawa M, Fukuda H, Nakano H, Abe S, Tateishi A, Kijima H, Yamazaki H, Tamaoki N, et al. Cell-retained isoforms of vascular endothelial growth factor (VEGF) are correlated with poor prognosis in osteosarcoma. Eur J Cancer. 1999; 35: 1089-93. doi: 10.1016/S09598049(99)00073-8.

22. Duque JL, Loughlin KR, Adam RM, Kantoff PW, Zurakowski D, Freeman MR. Plasma levels of vascular endothelial growth factor are increased in patients with metastatic prostate cancer. Urology. 1999; 54: 523-7. doi: 10.1016/S0090-4295(99)00167-3.

23. Calbet Jé AL. Chronic hypoxia increases blood pressure and noradrenaline spillover in healthy humans. J Physiol. 2003; 551: 379-86. doi: 10.1113/jphysiol.2003.045112.

24. Vaupel P, Mayer A. Hypoxia in cancer: significance and impact on clinical outcome. Cancer Metastasis Rev. 2007; 26: 225-39. doi: 10.1007/s10555-007-9055-1.

25. Pugh CW, Ratcliffe PJ. Regulation of angiogenesis by hypoxia: role of the HIF system. Nat Med. 2003; 9: 677-84. doi: 10.1038/nm0603-677.

26. Thom R, Rowe GC, Jang C, Safdar A, Arany Z. Hypoxic Induction of Vascular Endothelial Growth Factor (VEGF) and Angiogenesis in Muscle by Truncated Peroxisome Proliferator-activated Receptor $\gamma$ Coactivator (PGC)-

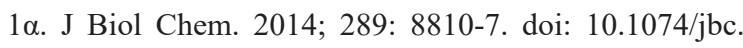
M114.554394.

27. Moeller BJ, Dewhirst MW. HIF-1 and tumour radiosensitivity. Br J Cancer. 2006; 95: 1-5. doi: 10.1038/ sj.bjc.6603201.

28. Moeller BJ, Cao Y, Li CY, Dewhirst MW. Radiation activates HIF-1 to regulate vascular radiosensitivity in tumors: role of reoxygenation, free radicals, and stress granules. Cancer Cell. 2004; 5: 429-41. doi: 10.1016/
S1535-6108(04)00115-1.

29. Bjork-Eriksson T, West C, Karlsson E, Mercke C. Tumor radiosensitivity (SF2) is a prognostic factor for local control in head and neck cancers. Int J Radiat Oncol Biol Phys. 2000; 46: 13-9. doi: 10.1016/S0360-3016(99)00373-9.

30. Freudlsperger C, Horn D, Weissfuss S, Weichert W, Weber KJ, Saure D, Sharma S, Dyckhoff G, Grabe N, Plinkert P, Hoffmann J, Freier K, Hess J. Phosphorylation of AKT(Ser473) serves as an independent prognostic marker for radiosensitivity in advanced head and neck squamous cell carcinoma. Int J Cancer. 2015; 136: 2775-85. doi: 10.1002/ijc. 29328.

31. Zeng Q, Guo X, Li NW, Xiang YQ, Cao SM, Hong $\mathrm{MH}$. [Clinical characteristics and prognosis of aged nasopharyngeal carcinoma patients: a report of 313 cases]. [Article in Chinese]. Ai Zheng. 2008; 27: 289-94. doi: 10.3892/mco.2014.432.

32. Leu YS, Chang YF, Lee JC, Lo AC, Chen YJ, Chen HW. Prognosis of Nasopharyngeal Carcinoma in the Elderly is Worse than in Younger Individuals-Experience of a Medical Institute. International Journal of Gerontology. 2014; 8: 81-4. doi: 10.1016/j.ijge.2013.08.008.

33. Farias TP, Dias FL, Lima RA, Kligerman J, de Sa GM, Barbosa MM, Goncalves FB Jr. Prognostic factors and outcome for nasopharyngeal carcinoma. Arch Otolaryngol Head Neck Surg. 2003; 129: 794-9. doi: 10.1001/ archotol.129.7.794.

34. Wu S, Wang B, Chen L, Xiong S, Zhuang F, Huang X, Wang M, Huang Z. Clinical efficacy of propranolol in the treatment of hemangioma and changes in serum VEGF, bFGF and MMP-9. Exp Ther Med. 2015; 10: 1079-83. doi: 10.3892/etm.2015.2650.

35. Krishna SM, James S, Balaram P. Expression of VEGF as prognosticator in primary nasopharyngeal cancer and its relation to EBV status. Virus Res. 2006; 115: 85-90. doi: 10.1016/j.virusres.2005.07.010.

36. Edge SB, Compton CC. The American Joint Committee on Cancer: the 7th edition of the AJCC cancer staging manual and the future of TNM. Ann Surg Oncol. 2010; 17: 1471-4. doi: 10.1245/s10434-010-0985-4.

37. Thompson L. World Health Organization classification of tumours: pathology and genetics of head and neck tumours. Ear Nose Throat J. 2006; 85: 74.

38. Randel A. AHA/ACC/ASH Release Guideline on the Treatment of Hypertension and CAD. Am Fam Physician. 2015; 92: 1023-30. 